\title{
Critical cluster composition from homogeneous nucleation data: application to water in carbon dioxide-nitrogen carrier gases
}

\author{
M. M. Campagna' ${ }^{1}$ - J. Hrubý' ${ }^{3}$ M. E. H. van Dongen ${ }^{2}$ D. M. J. Smeulders ${ }^{1}$
}

Received: 30 April 2021 / Revised: 28 June 2021 / Accepted: 19 July 2021 / Published online: 25 August 2021

(c) The Author(s) 2021

\begin{abstract}
Knowledge on critical cluster composition is important for improving the nucleation theory. Thus, homogeneous water nucleation experiments previously carried out in nitrogen and $0 \%, 5 \%, 15 \%$ and $25 \%$ of carbon dioxide (Campagna et al. 2020a, 2021) are analyzed. The tests were conducted at $240 \mathrm{~K}$ and $0.1 \mathrm{MPa}, 1 \mathrm{MPa}$ and $2 \mathrm{MPa}$. The observed nucleation rates are strongly dependent on supersaturation, pressure, temperature and mixture composition. These experimentally found dependencies can be used to derive the composition of critical clusters by means of the nucleation theorem. In this way, a macroscopic quantity, nucleation rate, reveals properties of critical clusters consisting of a few tens of molecules. Two novel methods are presented for the detailed application of the nucleation theorem. The first method extends to mixtures of $N>2$ components the approach used in literature for two components. The second method not only applies to $N>2$ mixtures in a more straightforward manner, but it can also be used for unary as well as for binary and multi-component nucleation cases. To the best of our knowledge, for the first time the critical cluster composition is computed for high pressure nucleation data of a vapor (here water) in mixtures of two carrier gases (here carbon dioxide-nitrogen). After a proper parameterization of the nucleation rate data, both methods consistently lead to the same critical nuclei compositions within the experimental uncertainty. Increasing pressure and carbon dioxide molar fraction at fixed supersaturation leads to a decrease in the water content of the critical cluster, while the adsorbed number of nitrogen and carbon dioxide molecules increases. As a consequence, the surface tension decreases. This outcome explains the observed increase in the nucleation rate with increasing pressure and carbon dioxide molar fraction at constant supersaturation.
\end{abstract}

\section{Graphic abstract}

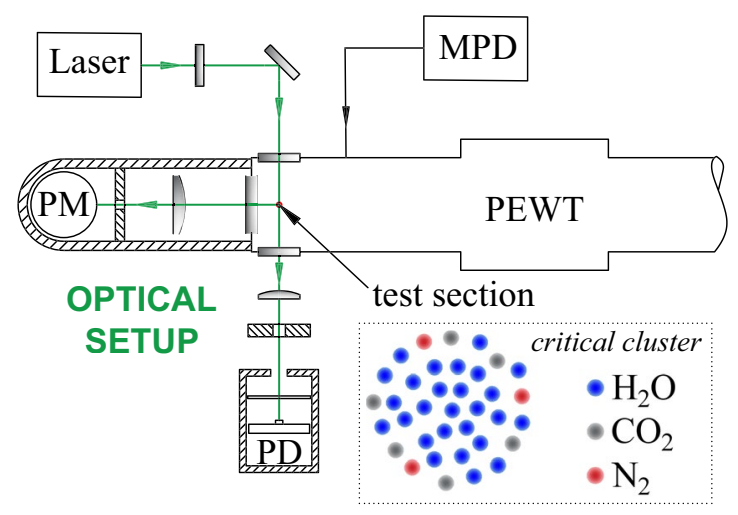

M. M. Campagna

M.M.Campagna@tue.nl

Extended author information available on the last page of the article

\section{Introduction}

Water condensation processes have important implications for a great variety of technological and climate related processes (Wyslouzil and Wölk 2016). Water condensation is 
considered homogeneous when the vapor-to-liquid transition takes place in absence of surfaces or foreign particles such as dust or ions. Under these circumstances, (Wilson and Thomson 1897) speculated that aggregates of water molecules, called nuclei, must act as condensation centers. Molecular clusters grow by collision with the free vapor molecules, while they also shrink by evaporation in a stochastic process. The probabilities of growth and decay are equal for the critical cluster. The number of supercritical clusters/droplets formed per unit time and volume is the so-called nucleation rate $J$. This quantity can be experimentally determined ( Campagna et al. 2020a, b, 2021). The homogeneous nucleation rate $J$ strongly depends on temperature $T$, pressure $p$, supersaturation $S$ of the condensing vapor and presence of carrier gases in the mixture. The supersaturation $S$ represents the driving force of the nucleation process and quantifies the current state deviation of the condensing component from its corresponding (same $p$ and $T$ ) phase equilibrium. A generally accepted expression for the nucleation rate $J$ is:

$J=K \exp \left(\frac{-W^{*}}{k T}\right)$

with $W^{*}$ the work of formation of a critical cluster, $K$ a preexponential factor, $k$ the Boltzmann constant, and $T$ the temperature.

According to the Classical Nucleation Theory (CNT) the size of a critical cluster can be estimated by means of the Gibbs-Thompson equation (Becker and Döring (1935)). This approach relies on the capillarity approximation, which assigns macroscopic surface energy properties to small clusters (typically some tens of molecules). In addition, the classical model does not account for the presence of carrier gas molecules at the cluster. As a result, the CNT does not lead to a satisfactory agreement with experiments. In Oxtoby and Kashchiev (1994), Oxtoby and Kashchiev proposed an alternative to the Gibbs-Thompson equation to evaluate the critical cluster content in a model-independent way: the nucleation theorem. They showed that, for a generic component $i$, its excess number of molecules in the critical cluster, $\Delta n_{i}^{*}$, relates to the partial derivative of the work of critical cluster formation $W^{*}$ as follows:

$\left.\frac{\partial W^{*}}{\partial \mu_{\mathrm{i}}^{\mathrm{g}}}\right|_{\mu_{j \neq i}^{\mathrm{g}}, T}=-\Delta n_{\mathrm{i}}^{*} \quad$ for $\mathrm{i}=1,2, . ., \mathrm{N}$,

with $\mu_{i}^{g}$ the chemical potential of component $i$ in its gaseous phase and $N$ the number of components in the mixture. The excess number $\Delta n_{i}^{*}$ is defined as the number of molecules in the critical cluster minus the number of molecules in the gas phase occupying the same volume of the cluster.

Combining Eqs. 1 and 2 enables to relate the excess number of molecules in the critical cluster to the measured nucleation rates $J$ at different pressure $p$, supersaturation $S$ and carrier gas molar fractions. For the present work, the condensing component is denoted as $i=1$, while the other components are not supersaturated. These components can enter the cluster by means of dissolution and adsorption.

Several examples can be found in literature on the analysis of the cluster composition by exploiting the nucleation theorem (Kashchiev 1982; Oxtoby and Laaksonen 1995; Luijten et al. 1998, 1999; Wölk and Strey 2001; Kalikmanov et al. 2007; Holten and van Dongen 2010). At high pressure $(>0.2 \mathrm{MPa}$ ), only few studies can be found in literature on the analysis of the critical cluster composition for water-carrier gas mixtures (Luijten et al. 1999; Holten and van Dongen 2010). Luijten et al. (1999) analyzed water-helium, water-nitrogen and n-nonane-methane mixtures at $240 \mathrm{~K}$ and $1 \mathrm{MPa}, 2.5 \mathrm{MPa}$ and $4 \mathrm{MPa}$. Using the supersaturation and pressure dependence of the experimental nucleation rates, they estimated the cluster composition for their binary mixtures (vapor in a single carrier gas). Following the same approach as Luijten et al. (1999), water-carbon dioxide-methane mixtures at $235 \mathrm{~K}$ and $1 \mathrm{MPa}$ were analyzed by Holten and van Dongen (2010). In this case, only the number of water and methane molecules in the critical cluster was determined.

The present work aims to cope with the lack of research studies on the critical cluster composition for mixtures of water in multiple carrier gas environments $(N>2)$ at high pressures. Providing information on the critical cluster composition is of paramount importance for improving the existing nucleation theory. For this purpose, two methods based on the nucleation theorem will be presented. With the first method, the approach generally used in literature for systems with two components is extended to mixtures with $(\mathrm{N}>2)$ components (Sect. 3.1). In addition, a more straightforward method to determine the critical cluster composition will be provided (Sect. 3.2), which can be used in the case of unary as well as multi-component nucleation. Both methods will be applied to the experimental nucleation data of water-carbon dioxide-nitrogen mixtures at $240 \mathrm{~K}^{1}$ and various pressures $(0.1 \mathrm{MPa}, 1 \mathrm{MPa}$ and $2 \mathrm{MPa})$ and carbon dioxide molar fractions $(0 \%, 5 \%, 15 \%$ and $25 \%)$ previously published by the authors (Campagna et al. 2020a, 2021). After careful

\footnotetext{
1 The Pulse Expansion Wave Tube (PEWT) allows to study homogeneous nucleation by experimental means at temperatures ranging from $220 \mathrm{~K}$ to $260 \mathrm{~K}$ ( Campagna et al. 2020b). The temperature range is limited to avoid the formation of ice crystals (for $\mathrm{T}<220 \mathrm{~K}$ ) and by PEWT gas dynamics constraints (for T $<260 \mathrm{~K}$ ). Within this temperature range, the choice of working at the intermediate temperature of $240 \mathrm{~K}$ is driven by the temperature conditions used in literature (Luijten et al. 1999; Holten and van Dongen 2010; Fransen et al. 2014, 2015) and adopted as references for our previous publications ( Campagna et al. 2020a, b, 2021).
} 


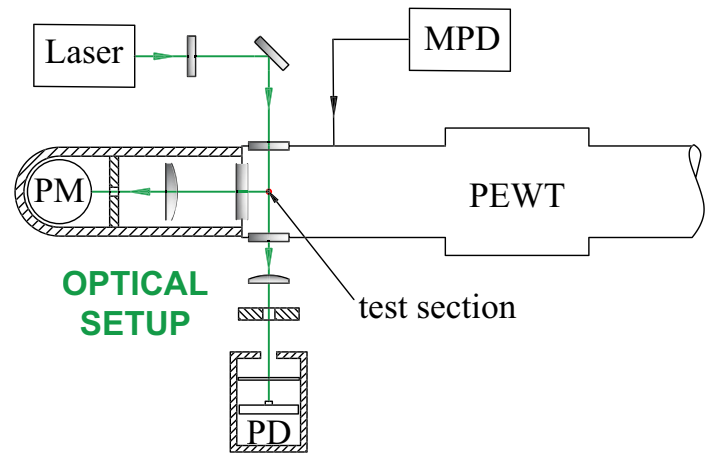

Fig. 1 Sketch of the experimental facility. The Pulse Expansion Wave Tube (PEWT) has a local widening at the low pressure section, enabling the application of the nucleation pulse method. The optical setup is placed at the test section and consists of a linearly polarized laser light with a wavelength of $532 \mathrm{~nm}$, a photodiode PD and a photomultiplier PM. The two pressure transducers are placed at the test section wall. The mixture preparation device is denoted as MPD

parameterization of the nucleation data (Eqs. 9 and 10), the cluster composition will be deduced for these experimental conditions (Sect. 4) by exploiting the nucleation rate dependence on supersaturation, pressure and carbon dioxide molar fraction (Method 1) and on the fugacities (Method 2).

\section{Experimental methodology overview}

The experimental data (Campagna et al. 2020a, 2021) used for the analysis of the present work were carried out with the Pulse Expansion Wave Tube (PEWT). A schematic of the facility is reported in Fig. 1 (more geometrical details in Campagna et al. 2020b). The PEWT is a special shock tube ( Looijmans and van Dongen 1997) implementing the nucleation pulse method (Peters 1983). This technique enables an effective decoupling of nucleation and droplet growth phenomena, which generates an almost monodisperse cloud of growing clusters/droplets (Campagna et al. 2020b). The number of critical clusters per unit of time and volume $J$ (nucleation rate) is experimentally determined as $J=n_{d} / \Delta t$, with $n_{d}$ the droplet number density and $\Delta t$ the nucleation pulse duration. The quantity $n_{d}$ is measured via a dedicated optical setup placed at the PEWT test section as sketched in Fig. 1. It consists of a 100:1 linearly polarized laser beam with a wavelength of $532 \mathrm{~nm}$, a photodiode PD and a photomultiplier PM. By means of the optical setup, Constant Angle Mie Scattering CAMS (Wagner and Strey 1981; Wagner 1985; Strey et al. 1994) is applied and $n_{d}$ can be determined. The nucleation pulse duration $\Delta t$ is obtained from the pressure signal, which is measured at the test section wall of the PEWT with two pressure transducers (Campagna et al. 2020b). The temperature $T$ at the pulse condition is determined by measuring the initial temperature of the test section wall and assuming isentropic expansion (Campagna et al. 2020a). A well-defined mixture composition $\left(y_{1}, y_{2}, y_{3}\right)$ is generated in the mixture preparation device (MPD) connected to the PEWT (see Fig. 1). In this way, knowing the thermodynamic conditions at the pulse ( $p$ and $T)$, the supersaturation can be determined as $S \approx y_{1} / y_{1, e q}$, $y_{1, e q}$ being dependent on $\left(p, T, y_{1}, y_{2}, y_{3}\right)$ (Campagna et al. 2021). A more extensive description of experimental methodology can be found in Campagna et al. (2020a, b, 2021)

For the analysis presented in this work, we use the homogeneous water nucleation experiments carried out in nitrogen and $0 \%, 5 \%, 15 \%$ and $25 \%$ of carbon dioxide, previously published by the authors (Campagna et al. 2020a, 2021). The tests were carried out at $240 \mathrm{~K}$ and three pressure conditions, $0.1 \mathrm{MPa}, 1 \mathrm{MPa}$ and $2 \mathrm{MPa}$. The results are shown in terms of nucleation rate $J$ and supersaturation $S$ in Fig. 4 (blue lines and symbols, with the standard uncertainty blue-shaded around the lines). It must be stressed that the experimental nucleation conditions were chosen such that the carrier gases were always in the undersaturated state. In fact, the saturation pressure of the pure carrier components was always larger than their partial pressure at the investigated conditions. In addition, the molar fractions of the carrier gases were so large that any carrier component in the gas phase was in chemical equilibrium with the corresponding carrier gas in the cluster and the nucleation kinetics only depended on impingement/evaporation of the condensing component molecules. In Luijten and van Dongen (1999), Luijten and van Dongen showed that nucleation phenomena of condensable vapors in a carrier gas can be considered as unary nucleation if $x_{g, e q}<<(S-1) / S$, with $x_{g, e q}$ the dissolved gas fraction in the bulk liquid. With a maximum carrier gas solubility $x_{\mathrm{CO}_{2}, e q}<0.038$ in our experiments (see supplementary material of Campagna et al. 2021), this condition was always fulfilled. For carrier gases with a much larger $x_{g, e q}$, the full binary nucleation model applies (Looijmans et al. 1995). Thus, we consider the nucleation phenomena analyzed in the present work as essentially unary, with water the only (supersaturated) component providing the driving force for the nucleation process.

\section{Application of the nucleation theorem to mixtures of $\boldsymbol{N}$ components at constant temperature}

For a mixture with $N$ components at constant temperature $T$, the nucleation rate $J$ and the work of critical cluster formation $W^{*}$ depend on a set of $N$ independent thermodynamic variables: $\left(\mu_{1}^{g}, \mu_{2}^{g}, \mu_{3}^{g}, \ldots, \mu_{N}^{g}\right)$ or equivalently $\left(S, p, y_{2}\right.$, $\left.\ldots, y_{N-1}\right)$. The partial derivative of $J$ with respect to these variables can be used in combination with the nucleation 
theorem in Eq. 2 to compute the $N$ unknowns $\left(\Delta n_{1}^{*}, \Delta n_{2}^{*}\right.$, $\left.\Delta n_{3}^{*}, \ldots, \Delta n_{N}^{*}\right)$.

The dependence of $J$ and $W^{*}$ on $S$ and $p$, in combination with the nucleation theorem, has been explored in literature to determine the cluster composition for mixtures with a maximum of two components, one of which being the condensing component (Luijten et al. 1999; Kalikmanov et al. 2007; Holten and van Dongen 2010). We will extend this procedure to a mixture of $N>2$ components in subsection 3.1 by exploiting the $J$ and $W^{*}$ dependence on $(S$, $\left.p, y_{2}, \ldots, y_{N-1}\right)$. Hereafter, this method will be denoted as Method 1. In addition, we will present in subsection 3.2 a novel methodology, based on the $J$ and $W^{*}$ dependence on the $N$ fugacities $\left(\mathcal{F}_{1}^{g}, \mathcal{F}_{2}^{g}, \mathcal{F}_{3}^{g}, \ldots, \mathcal{F}_{N}^{g}\right)$. Hereafter, this method will be referred to as Method 2. Such method will be shown to be more straightforward and easily applicable to multicomponent nucleation cases.

\subsection{Method 1}

We now consider the dependence of nucleation rate $J$ and work of cluster formation $W^{*}$ on the set of $N$ independent variables $\left(S, p, y_{2}, \ldots, y_{N-1}\right)$. The expression of the partial derivative of $J$ with respect to $S$ can be obtained by employing Eq. 1 and the chain rule as follows

$$
\begin{aligned}
\left.\frac{\partial \ln J}{\partial \ln S}\right|_{p, y_{2}, \ldots, y_{\mathrm{N}-1}} & =-\left.\left.\frac{1}{k T} \sum_{\mathrm{i}=1}^{\mathrm{N}} \frac{\partial W^{*}}{\partial \mu_{\mathrm{i}}^{\mathrm{g}}}\right|_{\mu_{j \neq i}^{\mathrm{g}}} \frac{\partial \mu_{\mathrm{i}}^{\mathrm{g}}}{\partial \ln S}\right|_{p, y_{2}, \ldots, y_{\mathrm{N}-1}}+1 \\
& =\left.\frac{1}{k T} \sum_{\mathrm{i}=1}^{\mathrm{N}} \Delta n_{\mathrm{i}}^{*} \frac{\partial \mu_{\mathrm{i}}^{\mathrm{g}}}{\partial \ln S}\right|_{p, y_{2}, \ldots, y_{\mathrm{N}-1}}+1,
\end{aligned}
$$

with the second equality obtained by substituting the nucleation theorem from Eq. 2. Note that $\partial \ln K / \partial \ln S=1$ in Eq. 3 for the following reason. In order to express the pre-exponential factor $K$, the kinetic model of nucleation needs to be considered. The impingement rate of vapor molecules is proportional to vapor concentration. Thus, $K$ is proportional to $S$.

Analogously to Eq. 3, $J$ can be derived with respect to $p$ leading to

$$
\begin{aligned}
\left.\frac{\partial \ln J}{\partial \ln p}\right|_{S, y_{2}, \ldots, y_{\mathrm{N}-1}} & =-\left.\left.\frac{p}{k T} \sum_{\mathrm{i}=1}^{\mathrm{N}} \frac{\partial W^{*}}{\partial \mu_{\mathrm{i}}^{\mathrm{g}}}\right|_{\mu_{j \neq i}^{\mathrm{g}}} \frac{\partial \mu_{\mathrm{i}}^{\mathrm{g}}}{\partial p}\right|_{S, y_{2}, \ldots, y_{\mathrm{N}-1}} \\
& =\left.\frac{p}{k T} \sum_{\mathrm{i}=1}^{\mathrm{N}} \Delta n_{\mathrm{i}}^{*} \frac{\partial \mu_{\mathrm{i}}^{\mathrm{g}}}{\partial p}\right|_{S, y_{2}, \ldots, y_{\mathrm{N}-1}},
\end{aligned}
$$

where $\partial \ln K / \partial \ln p \approx 0$. In order to justify the latter approximation, it must be considered that the expression of $K$ as a function of $\left(p, y_{2}, \ldots, y_{\mathrm{N}-1}\right)$ at constant $S$ depends on the kinetic model. We employed the kinetic model in the form of

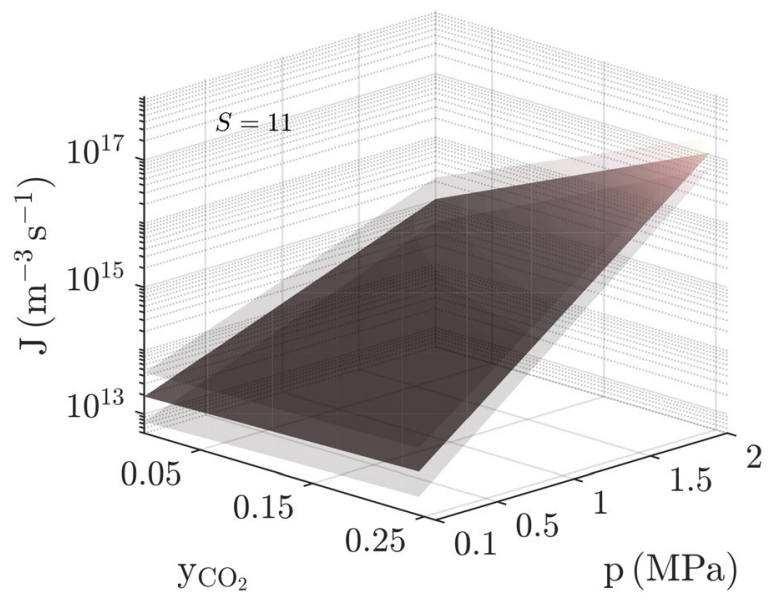

Fig. 2 3D view of $J$ parameterized as a function of $\left(S, p, \mathrm{y}_{\mathrm{CO} 2}\right.$, represented here for $S=11$. The standard uncertainty is also reported as thinner upper and lower layers

Courtney (Courtney 1961; Wilemski 1995) extended to the case of non-ideal mixtures (Campagna et al. 2020a). For our conditions, $\partial \ln K / \partial \ln p$ has been found to be much smaller than the uncertainty of $\partial \ln J / \partial \ln p$. Thus, $\partial \ln K / \partial \ln p$ can be safely neglected in deriving Eq. 4.

At this point, two linear Eqs. 3 and 4 are available with $N$ unknowns to be obtained. The remaining $(N-2)$ equations to close the system are given by the $(N-2)$ derivatives of $J$ with respect to each of the molar fractions $\left(y_{2}\right.$, $\left.\ldots, y_{\mathrm{q}}, \ldots, y_{\mathrm{N}-1}\right)$ as

$$
\begin{aligned}
\left.\frac{\partial \ln J}{\partial y_{q}}\right|_{S, p, y_{j \neq 11, q, N\}}}= & -\left.\left.\frac{1}{k T} \sum_{i=1}^{N} \frac{\partial W^{*}}{\partial \mu_{i}^{g}}\right|_{\mu_{j \neq i}^{g}} \frac{\partial \mu_{i}^{g}}{\partial y_{q}}\right|_{S, p, y_{j \neq 1, q, N\}}} \\
& =\left.\frac{1}{k T} \sum_{i=1}^{N} \Delta n_{i}^{*} \frac{\partial \mu_{i}^{g}}{\partial y_{q}}\right|_{S, p, y_{j \neq 11, q, N\}}},
\end{aligned}
$$

for $q=2,3, \ldots,(N-1)$. Note that $\partial \ln K / \partial y_{\mathrm{q}}$ is neglected in Eq. 5 for the same reason given for neglecting $\partial \ln K / \partial p$.

The left-hand sides of Eqs. 3-5 can be directly obtained from the experimental nucleation rates $J$ as a function of $\left(S, p, y_{2}, \ldots, y_{\mathrm{N}-1}\right)$. For our practical applications $(N=3)$, details will be given in Sec. 4 (see Eqs. 9 and Figs. 2 and 4).

Finally, in order to determine $\left(\Delta n_{1}^{*}, \Delta n_{2}^{*}, \Delta n_{3}^{*}, \ldots, \Delta n_{\mathrm{N}}^{*}\right)$ from Eqs. 3-5, each of the chemical potentials $\left(\mu_{1}^{\mathrm{g}}, \mu_{2}^{\mathrm{g}}, \mu_{3}^{\mathrm{g}}, \ldots, \mu_{\mathrm{N}}^{\mathrm{g}}\right)$ needs to be differentiated with respect to $\left(S, p, y_{2}, \ldots, y_{\mathrm{N}-1}\right)$. Details are given in Appendix 1.

\subsection{Method 2}

The definition of fugacity $\mathcal{F}_{i}^{g}$ (see Eq. 17) leads to $d \mu_{\mathrm{i}}^{\mathrm{g}}=k T d \ln \mathcal{F}_{\mathrm{i}}^{g}$ and Eq. 2 can be written as 


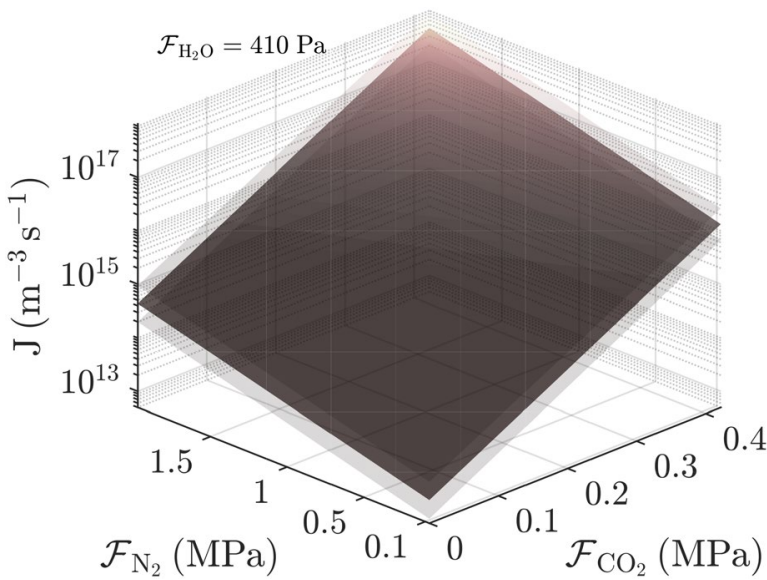

Fig. 3 3D view of $J$ parameterized as a function of $\mathcal{F}_{\mathrm{H}_{20}}^{g} \mathcal{F}_{\mathrm{CO}_{2}}^{g} \mathcal{F}_{N_{2}}^{g}$ ), represented here for $\mathcal{F}_{\mathrm{H}_{2 O}}^{g}=410 \mathrm{~Pa}$. The standard uncertainty is also reported as thinner upper and lower layers

$\left.\frac{\partial W^{*}}{\partial \mu_{\mathrm{i}}^{\mathrm{g}}}\right|_{\mu_{j \neq i}^{\mathrm{g}}}=\left.\frac{1}{k T} \frac{\partial W^{*}}{\partial \ln \mathcal{F}_{\mathrm{i}}^{g}}\right|_{\mathcal{F}_{j \neq i}^{g}}=-\Delta n_{\mathrm{i}}^{*}$.

We now consider the $J, K$ and $W^{*}$ dependence on $\left(\mathcal{F}_{1}^{g}, \mathcal{F}_{2}^{g}\right.$, $\left.\mathcal{F}_{3}^{g}, \ldots, \mathcal{F}_{\mathrm{N}}^{g}\right)$. From Eq. 1, the partial derivative of $J / K$ with respect to $\mathcal{F}_{\mathrm{i}}^{g}$ leads to

$\left.\frac{\partial \ln (J / K)}{\partial \ln \mathcal{F}_{i}}\right|_{\mathcal{F}_{j \neq i}}=-\left.\frac{1}{k T} \frac{\partial W^{*}}{\partial \ln \mathcal{F}_{i}^{g}}\right|_{\mathcal{F}_{j \neq i}^{g}}$.

Finally, by employing the nucleation theorem in the form of Eq. 6, we obtain that

$\left.\frac{\partial \ln (J / K)}{\partial \ln \mathcal{F}_{i}}\right|_{\mathcal{F}_{i \neq i}}=\Delta n_{i}^{*}$ for $i=1,2, \ldots, N$,

where, analogously to Method 1 (Sec. 3.1), it can be considered $\partial \ln K / \partial \ln \mathcal{F}_{1}^{g} \approx 1$ and $\partial \ln K / \partial \ln \mathcal{F}_{\mathrm{i}}^{g} \approx 0$ for $i=2,3, . ., N$.

Equation 8 enables a straightforward derivation of the cluster composition $\left(\Delta n_{1}^{*}, \Delta n_{2}^{*}, \Delta n_{3}^{*}, \ldots, \Delta n_{\mathrm{N}}^{*}\right)$ from the experimental $J$ data expressed as a function of the corresponding $\left(\mathcal{F}_{1}^{g}\right.$, $\left.\mathcal{F}_{2}^{g}, \mathcal{F}_{3}^{g}, \ldots, \mathcal{F}_{\mathrm{N}}^{g}\right)$. In addition, Eq. 8 can be directly applied to multi-component as well as to unary nucleation cases, which makes this methodology more general than Method 1. For our practical applications $(N=3)$, details will be given in Sect. 4 (see Eqs. 10 and Figs. 3 and 5).

\section{Critical cluster composition for water- carbon dioxide-nitrogen mixtures at $\mathbf{2 4 0}$ K}

The cluster composition is determined for mixtures of water in nitrogen with $0 \%, 5 \%, 15 \%$ and $25 \%$ of carbon dioxide, at $240 \mathrm{~K}$ and $0.1 \mathrm{MPa}, 1 \mathrm{MPa}$ and $2 \mathrm{MPa}$ (Campagna et al. 2020a, 2021). The analysis is based on the two methods derived in Sects. 3.1 and 3.2, applied to the case of three components $(N=3)$, where the unknowns are the excess number of water $\Delta n_{\mathrm{H}_{2} \mathrm{O}}^{*}$, carbon dioxide $\Delta n_{\mathrm{CO}_{2}}^{*}$ and nitrogen $\Delta n_{\mathrm{N}_{2}}^{*}$. Note that, hereafter, the subscript for the condensing component " 1 " will be assigned to water, " 2 " to carbon dioxide and " 3 " to nitrogen.

In order to determine $\Delta n_{\mathrm{H}_{20}}^{*}, \Delta n_{\mathrm{CO}_{2}}^{*}$ and $\Delta n_{\mathrm{N}_{2}}^{*}$, the calculation of the partial derivatives given in Eqs. 3-5 are required: $\partial \ln J / \partial \ln S, \partial \ln J / \partial \ln p$ and $\partial \ln J / \partial y_{\mathrm{CO}_{2}}$ (Method 1). For Method 2, determining the excess numbers of water, carbon dioxide and nitrogen requires the following partial derivatives to be computed from Eq. 8: $\partial \ln J / \ln \mathcal{F}_{\mathrm{H}_{2} \mathrm{O}}^{g}$, $\partial \ln J / \partial \ln \mathcal{F}_{\mathrm{CO}_{2}}^{g}$ and $\partial \ln J / \partial \ln \mathcal{F}_{\mathrm{N}_{2}}^{g}$. To this end, we parameterize $J$ as a function $\left(S, p, \mathrm{y}_{\mathrm{CO} 2}\right.$ and as a function of $\left(\mathcal{F}_{\mathrm{H}_{2} \mathrm{O}}^{g}\right.$, $\mathcal{F}_{\mathrm{CO}_{2}}^{g}, \mathcal{F}_{\mathrm{N}_{2}}^{g}$ ) to the experimental data. The following expressions are found to efficiently describe the data set for any experimental condition investigated in the present work (see Figs. 4 and 5).

- Method 1

$\ln J=A_{1}+\frac{C_{1}}{(\ln S)^{2}}$

where

$A_{1}=a_{10}+a_{12} p+a_{13} p y_{C O_{2}}$

$C_{1}=c_{10}+c_{12} p+c_{13} p y_{\mathrm{CO}_{2}}$

with $a_{10}=73.4, a_{12}=-4.39 \cdot 10^{-8}, a_{13}=-1.42 \cdot 10^{-5}$, $c_{10}=-247, c_{12}=1.24 \cdot 10^{-5}, c_{13}=1.36 \cdot 10^{-4}$, for $J$ in $\mathrm{m}^{-3} \mathrm{~s}^{-1}, p$ in $\mathrm{Pa}$ and $S$ non-dimensional.

In Fig. 2, the function $J\left(S, p, y_{\mathrm{CO}_{2}}\right)$ as from Eqs. 9 is represented for $S=11$. 
Table 1 Method 1 versus Method 2: excess numbers at the experimental conditions analyzed in the present work for $240 \mathrm{~K}$. The data reported here refer to $S=11$ for Method 1 and to $\mathscr{F}_{\mathrm{H}_{2} \mathrm{O}}^{g}=410 \mathrm{~Pa}$ for Method 2 . These two reference values are the only $S$ and $\mathscr{F}_{\mathrm{H}_{2} \mathrm{O}}^{g}$ in common to all $p$ and $\mathrm{y}_{\mathrm{CO} 2}$ conditions

\begin{tabular}{|c|c|c|c|c|c|c|c|}
\hline \multirow[t]{2}{*}{$\mathrm{yCO}_{2}$} & \multirow[t]{2}{*}{$\mathrm{p}(\mathrm{MPa})$} & \multicolumn{3}{|l|}{ Method 1} & \multicolumn{3}{|l|}{ Method 2} \\
\hline & & $\Delta n_{\mathrm{H}_{2} \mathrm{O}}^{*}$ & $\Delta n_{\mathrm{CO}_{2}}^{*}$ & $\Delta n_{N_{2}}^{*}$ & $\Delta n_{\mathrm{H}_{2} \mathrm{O}}^{*}$ & $\Delta n_{\mathrm{CO}_{2}}^{*}$ & $\Delta n_{N_{2}}^{*}$ \\
\hline 0 & 0.10172 & $34.7 \pm 4.3$ & & $0.16 \pm 0.07$ & $30.9 \pm 4.2$ & & $0.17 \pm 0.08$ \\
\hline 0.05 & 0.10095 & $34.6 \pm 5.2$ & $0.09 \pm 0.02$ & $0.15 \pm 0.07$ & $30.8 \pm 5.2$ & $0.08 \pm 0.02$ & $0.16 \pm 0.08$ \\
\hline 0.15 & 0.10067 & $34.4 \pm 5.2$ & $0.25 \pm 0.05$ & $0.14 \pm 0.06$ & $30.7 \pm 5.3$ & $0.24 \pm 0.05$ & $0.14 \pm 0.07$ \\
\hline 0.25 & 0.10072 & $34.2 \pm 5.2$ & $0.41 \pm 0.09$ & $0.12 \pm 0.05$ & $30.6 \pm 4.4$ & $0.39 \pm 0.09$ & $0.13 \pm 0.06$ \\
\hline 0 & 1.0018 & $33.1 \pm 4.4$ & & $1.70 \pm 0.77$ & $30.4 \pm 4.6$ & & $1.69 \pm 0.82$ \\
\hline 0.05 & 1.0020 & $30.1 \pm 4.9$ & $0.80 \pm 0.20$ & $1.64 \pm 0.74$ & $30.0 \pm 4.3$ & $0.72 \pm 0.16$ & $1.61 \pm 0.78$ \\
\hline 0.15 & 0.9960 & $30.1 \pm 5.5$ & $2.11 \pm 0.48$ & $1.54 \pm 0.70$ & $29.0 \pm 5.1$ & $2.15 \pm 0.49$ & $1.43 \pm 0.69$ \\
\hline 0.25 & 1.0051 & $28.1 \pm 5.2$ & $3.28 \pm 0.73$ & $1.38 \pm 0.32$ & $28.1 \pm 5.0$ & $3.57 \pm 0.81$ & $1.28 \pm 0.62$ \\
\hline 0 & 2.0004 & $31.3 \pm 4.4$ & & $3.47 \pm 1.57$ & $29.9 \pm 4.8$ & & $3.34 \pm 1.62$ \\
\hline 0.05 & 1.9957 & $29.3 \pm 5.0$ & $1.48 \pm 0.34$ & $3.46 \pm 1.56$ & $29.1 \pm 4.7$ & $1.33 \pm 0.30$ & $3.17 \pm 1.54$ \\
\hline 0.15 & 2.0003 & $25.4 \pm 4.4$ & $3.90 \pm 0.90$ & $3.34 \pm 1.51$ & $27.4 \pm 4.4$ & $3.95 \pm 0.89$ & $2.85 \pm 1.38$ \\
\hline 0.25 & 2.0002 & $21.5 \pm 4.0$ & $6.33 \pm 1.63$ & $3.20 \pm 1.45$ & $25.8 \pm 4.0$ & $6.49 \pm 1.47$ & $2.53 \pm 1.23$ \\
\hline
\end{tabular}

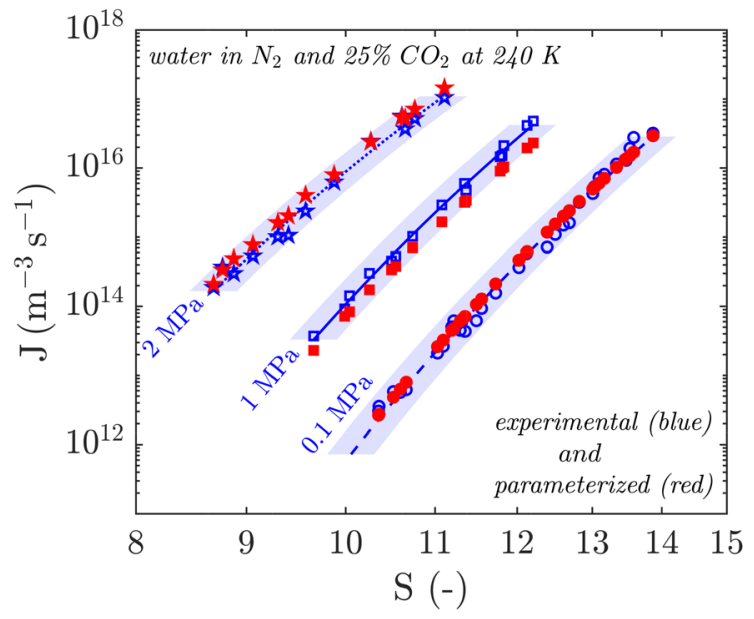

(a)

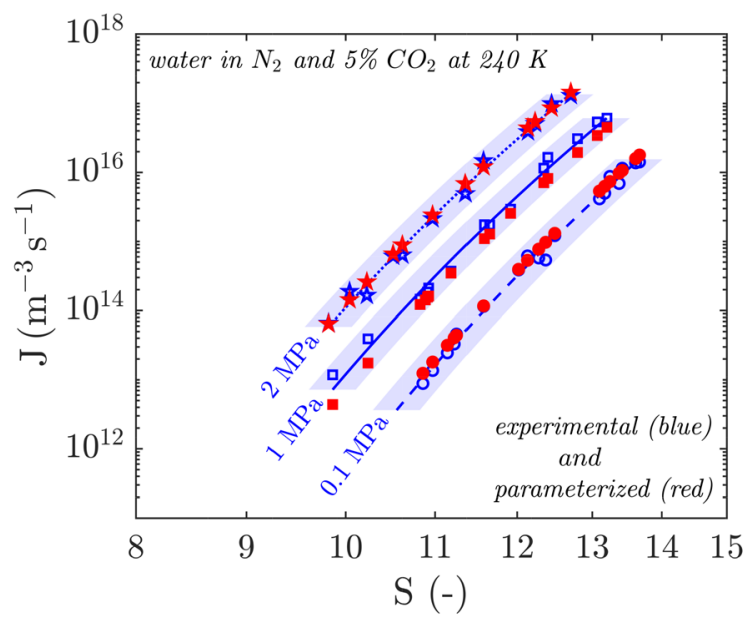

(c)

Fig. 4 Method 1: $J-S$ plots of the homogeneous water nucleation data (blue lines and symbols from Campagna et al. 2020a, 2021) carried out in nitrogen and 25\% (4a), 15\% (4b), 5\% (4c) and 0\% (4d) of carbon dioxide at $0.1 \mathrm{MPa}$ (dashed lines), $1 \mathrm{MPa}$ (solid lines) and 2

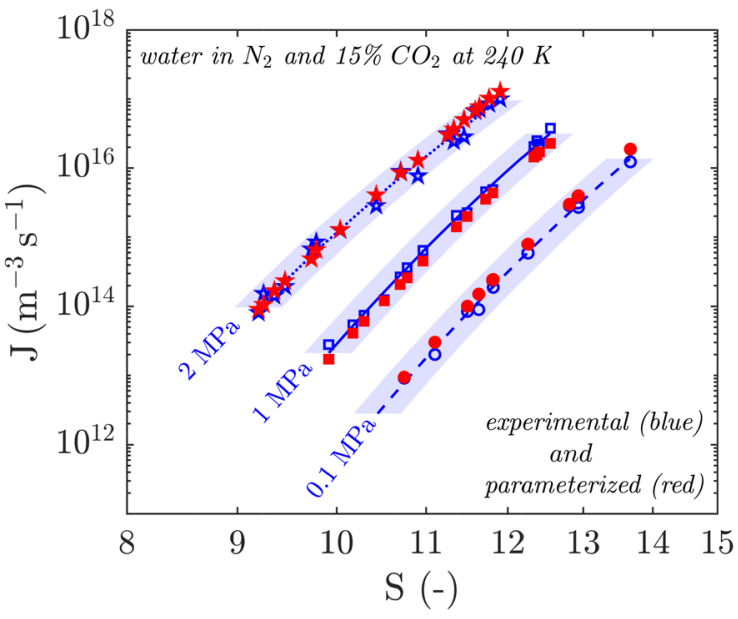

(b)

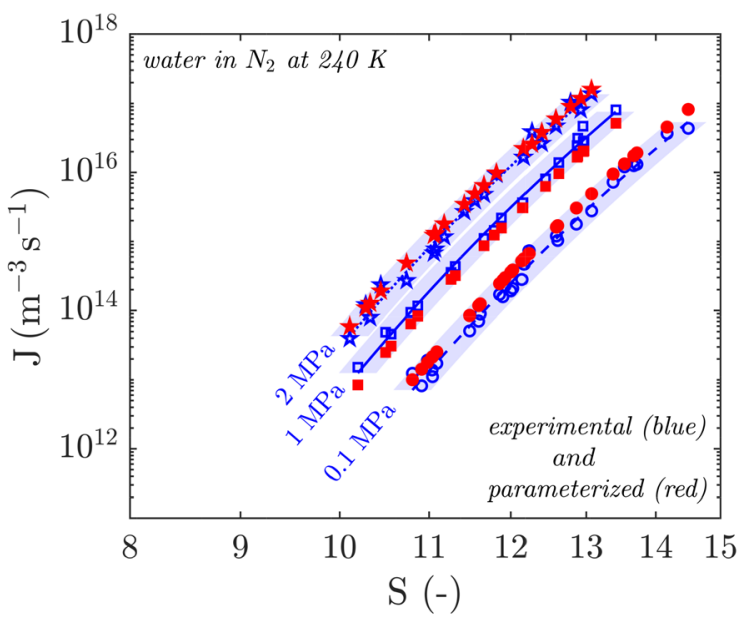

(d)

MPa (dotted lines). The standard uncertainty is blue-shaded around the lines. The experimental $J-S$ data are compared to the values of $J$ calculated from Eqs. 9 (red symbols) at the experimental $\left(S, p, \mathrm{y}_{\mathrm{CO} 2}\right.$ 


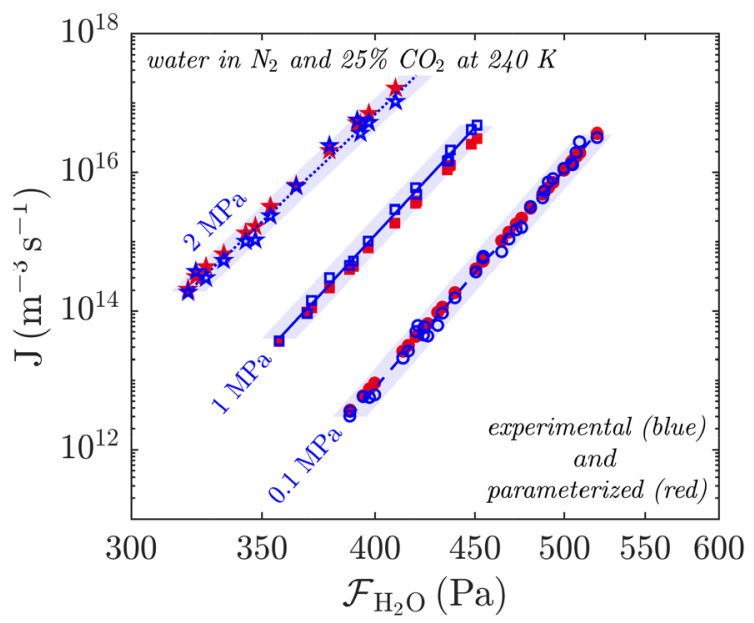

(a)

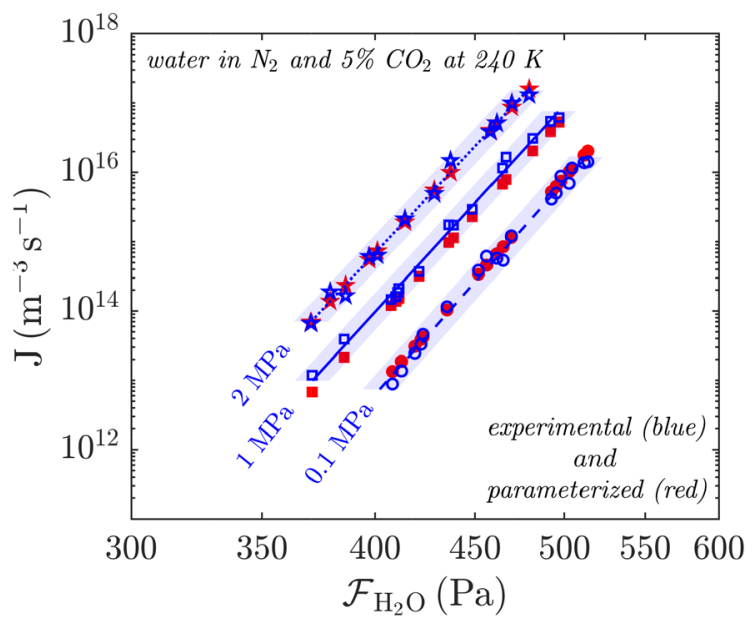

(c)

Fig. 5 Method 2: $J-\left(\mathcal{F}_{H_{2} O}^{g}\right.$ plots of the homogeneous water nucleation experiments (blue lines and symbols from Campagna et al. 2020a, 2021) carried out in nitrogen and 25\% (5a), 15\% (5b), 5\% (5c) and $0 \%$ (5d) of carbon dioxide at $0.1 \mathrm{MPa}$ (dashed lines), $1 \mathrm{MPa}$ (solid

- Method 2

$\ln J=A_{2}+C_{2} \ln \mathcal{F}_{\mathrm{H}_{20}}^{\mathrm{g}}$

where

$A_{2}=a_{20}+a_{22} \mathcal{F}_{\mathrm{CO}_{2}}^{\mathrm{g}}+a_{23} \mathcal{F}_{\mathrm{N}_{2}}^{\mathrm{g}}$

$C_{2}=c_{20}+c_{22} \mathcal{F}_{\mathrm{CO}_{2}}^{\mathrm{g}}+c_{23} \mathcal{F}_{\mathrm{N}_{2}}^{\mathrm{g}}$

with $a_{20}=162, a_{22}=7.97 \cdot 10^{-5}, a_{23}=4.90 \cdot 10^{-6}$, $c_{20}=31.9, c_{22}=-1.05 \cdot 10^{-5}, c_{23}=-5.10 \cdot 10^{-7}$, for $J$ in $\mathrm{m}^{-3} \mathrm{~s}^{-1}$ and $\mathcal{F}_{\mathrm{H}_{2} \mathrm{O}}^{g}, \mathcal{F}_{\mathrm{CO}_{2}}^{g}, \mathcal{F}_{\mathrm{N}_{2}}^{g}$ in Pa.

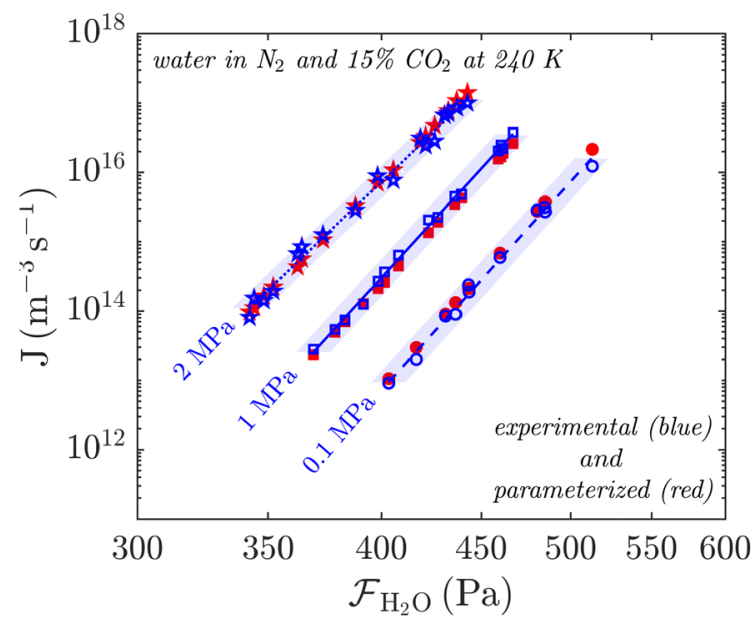

(b)

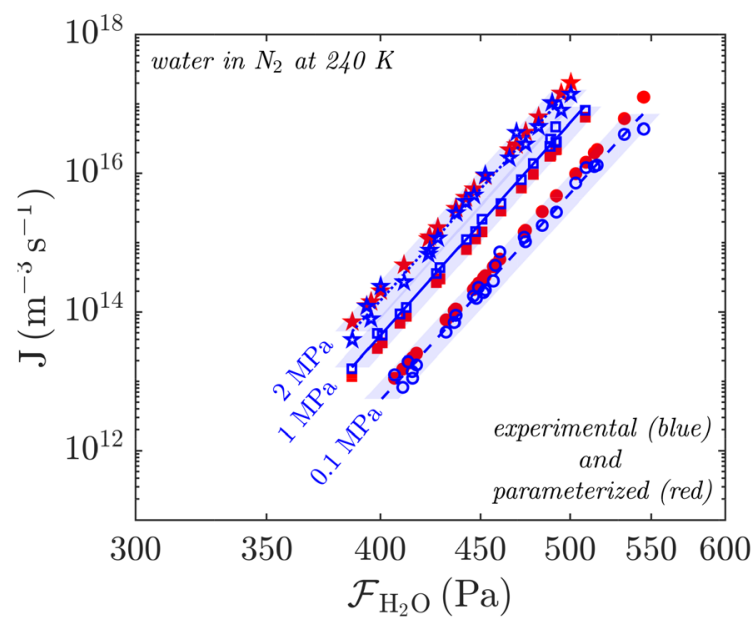

(d)

lines) and $2 \mathrm{MPa}$ (dotted lines). The standard uncertainty is blueshaded around the lines. The experimental $J-\left(\mathcal{F}_{\mathrm{H}_{2} \mathrm{O}}^{g}\right.$ data are compared to the values of $J$ calculated from Eqs. 10 (red symbols) at the experi$\operatorname{mental}\left(\mathcal{F}_{\mathrm{H}_{2} \mathrm{O}}^{g} \mathcal{F}_{\mathrm{CO}_{2}}^{g} \mathcal{F}_{\mathrm{N}_{2}}^{g}\right.$

In Fig. 3, the function $J\left(\mathcal{F}_{\mathrm{H}_{2 \mathrm{O}}}^{g}, \mathcal{F}_{\mathrm{CO}_{2}}^{g}, \mathcal{F}_{\mathrm{N}_{2}}^{g}\right)$ as from Eqs. 10 is represented for $\mathcal{F}_{\mathrm{H}_{20}}^{g}=410 \mathrm{MPa}$.

In this way, the partial derivatives of the nucleation rate $J$ can be determined.

Finally, $\Delta n_{\mathrm{H}_{20}}^{*}, \Delta n_{\mathrm{CO}_{2}}^{*}$ and $\Delta n_{\mathrm{N}_{2}}^{*}$ can be calculated and the outcomes for Method 1 and 2 are reported in Table 1 .

The results obtained with both methods show a good agreement within the uncertainty bands. This finding supports the validity of both methods, with Method 2 being more straightforward and easily applicable to unary as well as multi-component nucleation cases (Sect. 3). It must be noted that a small difference between the outcomes of the two methods can be observed. We argue that 
this difference can be attributed to the different ways with which the data are parameterized (see Eqs. 9 and Eqs. 10). However, the agreement between the two methods proves that the parameterizations are consistent. Given the equivalence of the two methods, we refer to Method 1 only.

At fixed carbon dioxide molar fraction $y_{\mathrm{CO}_{2}}$ and supersaturation $S, \Delta n_{\mathrm{H}_{2} \mathrm{O}}^{*}$ decreases with pressure, while $\Delta n_{\mathrm{CO}_{2}}^{*}$ and $\Delta n_{\mathrm{N}_{2}}^{*}$ increase (see Table 1). For $y_{\mathrm{CO}_{2}}=0.25, \Delta n_{\mathrm{H}_{2} \mathrm{O}}^{*}$ ranges form $34.2 \pm 5.2$ at $0.1 \mathrm{MPa}$ to $21.5 \pm 4.0$ at $2 \mathrm{MPa}$. On the other hand, $\Delta n_{\mathrm{CO}_{2}}^{*}$ and $\Delta n_{\mathrm{N}_{2}}^{*}$ increase with the pressure: $\Delta n_{\mathrm{CO}_{2}}^{*}$ vary from $0.41 \pm 0.09$ at $0.1 \mathrm{MPa}$ to $6.33 \pm 1.63$ at $2 \mathrm{MPa}$ and $\Delta n_{\mathrm{N}_{2}}^{*}$ ranges from $0.12 \pm 0.05$ at $0.1 \mathrm{MPa}$ to $3.20 \pm 1.45$ at $2 \mathrm{MPa}$. This behavior consistently repeats itself at smaller $y_{\mathrm{CO}_{2}}$, becoming less pronounced with decreasing the carbon dioxide molar fraction.

At fixed pressure $p$ and supersaturation $S, \Delta n_{\mathrm{H}_{20}}^{*}$ decreases with increasing $y_{\mathrm{CO}_{2}}$, while $\Delta n_{\mathrm{CO}_{2}}^{*}$ increase and $\Delta n_{\mathrm{N}_{2}}^{*}$ somewhat decreases (see Table 1). For $2 \mathrm{MPa}$, the values of $\Delta n_{\mathrm{H}_{2} \mathrm{O}}^{*}$ vary from $31.3 \pm 4.4$ for $y_{\mathrm{CO}_{2}}=0$ to $21.5 \pm 4.0$ for $y_{\mathrm{CO}_{2}}=0.25$. At $2 \mathrm{MPa}$, the $\Delta n_{\mathrm{CO}_{2}}^{*}$ values increase from $1.48 \pm 0.34$ for $y_{\mathrm{CO}_{2}}=0.05$ to $6.33 \pm 1.63$ for $y_{\mathrm{CO}_{2}}=0.25$. The values of $\Delta n_{\mathrm{N}_{2}}^{*}$ slightly decrease with increasing $y_{\mathrm{CO}_{2}}$ at $2 \mathrm{MPa}$. However, this small decrease remains well within the uncertainty bands (for both methods) and $\Delta n_{\mathrm{N}_{2}}^{*}$ can be considered approximately constant. The behavior observed at $2 \mathrm{MPa}$ is also found at $1 \mathrm{MPa}$ and $0.1 \mathrm{MPa}$, but with consistently bigger values of $\Delta n_{\mathrm{H}_{20}}^{*}$ and smaller $\Delta n_{\mathrm{CO}_{2}}^{*}$ and $\Delta n_{\mathrm{N}_{2}}^{*}$.

The microscopic picture given by the critical cluster composition in Table 1 provides important information. At constant supersaturation (the driving force of nucleation), increasing $p$ and $y_{\mathrm{CO}_{2}}$ facilitates the nucleation process as $J$ increases (see Fig. 4). This effect is due to more and more carrier gas molecules adsorbed at the cluster surface. Their increasing excess numbers with increasing $p$ and $y_{\mathrm{CO}_{2}}$ reduces the energy of formation available for the water molecules, which explains the smaller $\Delta n_{\mathrm{H}_{20}}^{*}$. The larger number of carrier gas molecules adsorbed $^{2}$ at the cluster surface causes a decrease in the surface tension, leading to the observed increase in $J$ with $p$ and $y_{\mathrm{CO}_{2}}$ (see Fig. 4). An estimate of the surface tension reduction for the analyzed nucleation condition was given in Campagna et al. (2021): with $y_{\mathrm{CO}_{2}}=0$, the surface tension decrease is of about $0.25 \%$ at $0.1 \mathrm{MPa}$ and $4.5 \%$ at $2 \mathrm{MPa}$; with $y_{\mathrm{CO}_{2}}=0.25$, the surface tension decrease is consistently larger and of about $1.4 \%$ at $0.1 \mathrm{MPa}$ and $19.1 \%$ at $2 \mathrm{MPa}$. This causes the

\footnotetext{
2 As demonstrated by Luijten and van Dongen (1999), the carrier gas bulk fractions are smaller than its equilibrium liquid fractions. Therefore, the carrier gas molecules at the critical cluster can be all considered as located at the surface.
}

experimental work of critical cluster formation to decrease and, as a consequence, the observed nucleation rates increase with $p$ and $y_{\mathrm{CO}_{2}}$. By increasing the pressure from $0.1 \mathrm{MPa}$ to $2 \mathrm{MPa}$, in fact, the magnitude of decrease in the critical work of cluster formation is $\sim 10 \%$ with $y_{\mathrm{CO}_{2}}=0$ and $\sim 36 \%$ with $y_{\mathrm{CO}_{2}}=0.25$.

It is worth mentioning that, even though $y_{\mathrm{CO}_{2}}<y_{\mathrm{N}_{2}}$ for any investigated condition, the presence of carbon dioxide at high pressure (1 MPa and $2 \mathrm{MPa}$ ) significantly increases $J$ with respect to the case of $y_{\mathrm{CO}_{2}}=0$. This is coherent with the much stronger effect that carbon dioxide has on the surface tension reduction, even for the conditions in which $\Delta n_{\mathrm{CO}_{2}}^{*}$ is smaller than or comparable to $\Delta n_{\mathrm{N}_{2}}^{*}$.

\section{Conclusions}

The nucleation theorem is used to deduce the cluster compositions at constant temperature $T$ by experimental means. Two novel methods (Method 1 and 2) are derived from the nucleation theorem for mixtures of $N>2$ components, with one being supersaturated (Sect. 3). The nucleation rate dependence on supersaturation, pressure and mixture composition is used in Method 1 (Sect. 3.1). This method extends to mixtures of $N>2$ components the approach used in literature for two components only (Kashchiev 1982; Oxtoby and Laaksonen 1995; Luijten et al. 1998, 1999; Wölk and Strey 2001; Kalikmanov et al. 2007; Holten and van Dongen 2010). Method 2 (Sect. 3.2) relies on the nucleation rate dependence on the $N$ fugacities. It can be used in case of unary as well as multi-component nucleation and it greatly simplifies the calculations with respect to the first method.

The two methods are applied to homogeneous water nucleation experiments in nitrogen and $0 \%, 5 \%, 15 \%$ and $25 \%$ of carbon dioxide, previously published by the authors (Campagna et al. 2020a, 2021). The experiments were carried out at $240 \mathrm{~K}$ and $0.1 \mathrm{MPa}, 1 \mathrm{MPa}$ and $2 \mathrm{MPa}$. As a result, the excess numbers of water, carbon dioxide and nitrogen are deduced for the whole set of analyzed experimental conditions (Table 1). To the best of our knowledge, for the first time the critical cluster composition is deduced for mixtures of water in more than one carrier gas at high pressure $(>0.2 \mathrm{MPa})$. After parameterization of the nucleation rate data (see Eqs. 9 and Eqs. 10), Methods 1 and 2 gave equivalent results in terms of critical cluster composition. This finding supports the validity of both methods and proves the used parameterizations to be consistent.

At constant carbon dioxide fractions, the excess number of water molecules decreases with increasing the pressure, while the excess number of carbon dioxide and nitrogen increase. At constant pressure, the water molecules in the critical cluster decrease with increasing the carbon dioxide 
content, while the excess number of carbon dioxide predictably increases. In this case, the nitrogen excess number remains approximately constant.

The microscopic overview that results from this analysis can be interpreted as follows. Increasing pressure and carbon dioxide fraction at constant supersaturation (the driving force of nucleation) increases the nucleation rate, meaning that the nucleation process is greatly facilitated ( $J$ increases). This is due to a larger number of carbon dioxide and nitrogen molecules adsorbed at the cluster surface. Adsorption phenomena cause a surface tension decrease, thus explaining the observed nucleation rate increase with pressure and carbon dioxide molar fraction (Campagna et al. 2020a, 2021). The pulse expansion wave tube experiments can thus successfully be used to unravel the composition of condensing clusters on the molecular scale, which is crucial for the advancement of the existing nucleation theory.

\section{Chemical potentials and partial derivatives}

In this section, we will give more details on the partial derivatives of the chemical potentials in Eqs. 3-5. To this end, each of the chemical potentials $\left(\mu_{1}^{\mathrm{g}}, \mu_{2}^{\mathrm{g}}, \mu_{3}^{\mathrm{g}}, \ldots, \mu_{\mathrm{N}}^{\mathrm{g}}\right)$ needs to be expressed as a function of $\left(S, p, y_{2}, \ldots, y_{\mathrm{N}-1}\right)$.

We start with the partial derivative of the chemical potentials $\left(\mu_{1}^{\mathrm{g}}, \mu_{2}^{\mathrm{g}}, \mu_{3}^{\mathrm{g}}, \ldots, \mu_{\mathrm{N}}^{\mathrm{g}}\right)$ to the logarithm of $S$ at constant $(p$, $\left.y_{2}, \ldots, y_{\mathrm{N}-1}\right)$ in Eq. 3. From the definition of supersaturation $S=\mathcal{F}_{1}^{\mathrm{g}} / \mathcal{F}_{1, \text { eq }}^{\mathrm{g}}$ (Campagna et al. 2020a),

$$
\begin{aligned}
\mu_{1}^{\mathrm{g}} & =\mu_{1, \mathrm{eq}}+k T \ln S \\
& =\mu_{1, \mathrm{~s}}+k T \ln x_{1, \mathrm{eq}}+\int_{p_{1, \mathrm{~s}}}^{p} v_{1}^{\ell} d p+k T \ln S,
\end{aligned}
$$

with $\mu_{1, \text { eq }}$ the chemical potential of the condensing component " 1 " with its gaseous and liquid phase at equilibrium, $x_{1, \mathrm{eq}}$ the liquid fraction of " 1 " at equilibrium, $\left(\int_{p_{1, s}}^{p} v_{1}^{\ell} d p\right)$ the so-called Poynting effect and with $\mu_{1, \mathrm{~s}}$ and $p_{1, \mathrm{~s}}$ the chemical potential and the pressure of the pure component " 1 " at saturation (Campagna et al. 2020a). It follows that

$\left.\frac{\partial \mu_{1}^{\mathrm{g}}}{\partial \ln S}\right|_{p, y_{2}, \ldots, y_{\mathrm{N}-1}}=k T$.

In addition, for $y_{1} \ll\left(y_{2}, y_{3}, . ., y_{\mathrm{N}}\right)$, it can be safely assumed that $\left(\mu_{2}^{\mathrm{g}}, \mu_{3}^{\mathrm{g}}, \ldots, \mu_{\mathrm{N}}^{\mathrm{g}}\right)$ are not influenced by the variations of $S$ due to the variation of $y_{1}$ at constant $T$ and $\left(p, y_{2}, . ., y_{\mathrm{N}-1}\right)$. Hence,

$$
\left.\frac{\partial \mu_{\mathrm{i}}^{\mathrm{g}}}{\partial \ln S}\right|_{p, y_{2}, \ldots, y_{\mathrm{N}-1}}=0 \quad \text { for } \mathrm{i}=2,3, . ., \mathrm{N} .
$$

Finally, Eq. 3 simplifies as follows $\left.\frac{\partial \ln (J / K)}{\partial \ln S}\right|_{p, y_{2}, \ldots, y_{\mathrm{N}-1}}=\Delta n_{1}^{*}$

which enables a straightforward calculation of $\Delta n_{1}^{*}$ from the experimental $J-S$ curves at constant $T$ and $\left(p, y_{2}, . ., y_{\mathrm{N}-1}\right)$.

The partial derivative of $\mu_{1}^{\mathrm{g}}$ with respect to $p$ in Eq. 4 and with respect to $\partial y_{\mathrm{q}}$ in Eq. 5 can be obtained from Eq. 11 as

$\left.\frac{\partial \mu_{1}^{\mathrm{g}}}{\partial p}\right|_{S, y_{2}, \ldots, y_{\mathrm{N}-1}}=v_{1}^{\ell}+\left.\frac{k T}{x_{1, \mathrm{eq}}} \frac{\partial x_{1, \mathrm{eq}}}{\partial p}\right|_{S, y_{2}, \ldots, y_{\mathrm{N}-1}}$

and

$\left.\frac{\partial \mu_{1}^{g}}{\partial y_{q}}\right|_{S, p, y_{j \neq\{1, q, N\}}}=\left.\frac{k T}{x_{1, e q}} \frac{\partial x_{1, e q}}{\partial y_{q}}\right|_{p, y_{j \neq 11, q, N\}}}$.

The partial derivative of the chemical potentials $\left(\mu_{1}^{\mathrm{g}}, \mu_{2}^{\mathrm{g}}, \mu_{3}^{\mathrm{g}}\right.$, ..,$\left.\mu_{\mathrm{N}}^{\mathrm{g}}\right)$ to the pressure $p$ at constant $\left(S, y_{2}, . ., y_{\mathrm{N}-1}\right)$ in Eq. 4 can be obtained as follows. We now introduce the correlation between chemical potential $\mu_{\mathrm{i}}^{\mathrm{g}}$ and fugacity $\mathcal{F}_{\mathrm{i}}^{g}$ for the generic $i^{\text {th }}$ component in its gaseous phase

$\mu_{\mathrm{i}}^{\mathrm{g}}=\mu_{\mathrm{i}, \text { ref }}\left(p_{\text {ref }}, T\right)+k T \ln \left[\frac{\mathcal{F}_{\mathrm{i}}^{g}}{\mathcal{F}_{\mathrm{i}, \text { ref }}\left(p_{\text {ref }}, T\right)}\right]$,

where $\mu_{\mathrm{i} \text {,ref }}$ and $\mathcal{F}_{\mathrm{i} \text {,ref }}$ are the chemical potential and the fugacity of the pure component $i$ at the temperature $T$ and an arbitrary reference pressure $p_{\text {ref }}$. Thus, at constant temperature $T$

$\left.\frac{\partial \mu_{\mathrm{i}}^{\mathrm{g}}}{\partial p}\right|_{S, y_{2}, \ldots, y_{\mathrm{N}-1}}=\left.\frac{k T}{\mathcal{F}_{\mathrm{i}}^{g}} \frac{\partial \mathcal{F}_{\mathrm{i}}^{g}}{\partial p}\right|_{y_{2}, \ldots, y_{\mathrm{N}-1}}$

and

$\left.\frac{\partial \mu_{\mathrm{i}}^{\mathrm{g}}}{\partial y_{\mathrm{q}}}\right|_{S, p, y_{\mathrm{j} \neq\{1, q, N\}}}=\left.\frac{k T}{\mathcal{F}_{\mathrm{i}}^{g}} \frac{\partial \mathcal{F}_{\mathrm{i}}^{g}}{\partial y_{\mathrm{q}}}\right|_{p, y_{j \neq\{1, q, N\}}}$,

with

$\mathcal{F}_{\mathrm{i}}^{g}=\phi_{\mathrm{i}} y_{\mathrm{i}} p$.

The parameter $\phi_{\mathrm{i}}$ in Eq. 20 denotes the fugacity coefficient, which can be defined by means of the virial EOS as

$\ln \phi_{i}=\left[2 \sum_{j=1}^{N} B_{i j} y_{j}-B\right] \frac{p}{R T}$,

where $B$ is the second virial coefficient of the mixture, defined as 


$$
B=\sum_{\mathrm{i}=1}^{\mathrm{N}} \sum_{\mathrm{j}=1}^{\mathrm{N}} y_{\mathrm{i}} y_{\mathrm{j}} B_{\mathrm{ij}} .
$$

In Eqs. 21 and 22, $B_{\text {ii }}$ denotes the second virial coefficient of the pure $i^{\text {th }}$ component and $B_{i j \neq i}$ stands for the second crossvirial coefficient of the $i$-th and $j$-th interacting components (Campagna et al. 2020a).

Acknowledgements J.H. acknowledges support from the Ministry of Education, Youth and Sports of the Czech Republic under OP RDE Grant No. CZ.02.1.01/0.0/0.0/16_019/0000753 "Research center for low carbon energy technologies" and institutional support RVO:61388998.

Open Access This article is licensed under a Creative Commons Attribution 4.0 International License, which permits use, sharing, adaptation, distribution and reproduction in any medium or format, as long as you give appropriate credit to the original author(s) and the source, provide a link to the Creative Commons licence, and indicate if changes were made. The images or other third party material in this article are included in the article's Creative Commons licence, unless indicated otherwise in a credit line to the material. If material is not included in the article's Creative Commons licence and your intended use is not permitted by statutory regulation or exceeds the permitted use, you will need to obtain permission directly from the copyright holder. To view a copy of this licence, visit http://creativecommons.org/licenses/by/4.0/.

\section{References}

Becker R, Döring W (1935) Kinetische behandlung der keimbildung in übersättigten dämpfen. Annalen der Physik 416(8), 719-752. https://doi.org/10.1002/andp.19354160806

Campagna MM, Hrubý J, van Dongen MEH, Smeulders DMJ (2020a) Homogeneous water nucleation: Experimental study on pressure and carrier gas effects. J Chem Phys 153(16):164303. https://doi. org/10.1063/5.0021477

Campagna MM, van Dongen MEH, Smeulders DMJ (2020b) Novel test section for homogeneous nucleation studies in a pulse expansion wave tube: experimental verification and gasdynamic $2 \mathrm{~d}$ numerical model. Exp Fluids 61(4):108. https://doi.org/10.1007/ s00348-020-02945-3

Campagna MM, Hrubý J, van Dongen MEH, Smeulders DMJ (2021) Homogeneous water nucleation in carbon dioxide-nitrogen mixtures: Experimental study on pressure and carrier gas effects. J. Chem. Phys. 154(15):154301.https://doi.org/10.1063/5.0044898

Courtney WG (1961) Remarks on homogeneous nucleation. J. Chem. Phys. 35:2249-2250. https://doi.org/10.1063/1.1732252

Fransen MALJ, Sachteleben E, Hrubý J, Smeulders DMJ (2014) On the growth of homogeneously nucleated water droplets in nitrogen: an experimental study. Exp Fluids 55(7):1780. https://doi.org/10. 1007/s00348-014-1780-y

Fransen MALJ, Hrubý J, Smeulders DMJ, van Dongen MEH (2015) On the effect of pressure and carrier gas on homogeneous water nucleation. J Chem Phys 142(16):164307. https://doi.org/10. $1063 / 1.4919249$

Holten V, van Dongen M (2010) Homogeneous water nucleation and droplet growth in methane and carbon dioxide mixtures at $235 \mathrm{~K}$ and 10 bar. J. Chem. Phys. 132:204504. https://doi.org/10.1063/1. 3432623
Kalikmanov V, Betting M, Bruining J, Smeulders D (2007) New developments in nucleation theory and their impact on natural gas separation. In: Proceedings of the SPE annual technical conference and exhibition, 11-14 November 2007, Anaheim, California, pp 11-14

Kashchiev D (1982) On the relation between nucleation work, nucleus size, and nucleation rate. J Chem Phys 76(10), 5098-5102. https:// doi.org/10.1063/1.442808

Looijmans KNH, van Dongen MEH (1997) A pulse-expansion wave tube for nucleation studies at high pressures. Exp Fluids 23:54-63. https://doi.org/10.1007/s003480050086

Looijmans KNH, Luijten CCM, Hofmans GCJ, van Dongen MEH (1995) Classical binary nucleation theory applied to the real mixture n-nonane/methane at high pressures. J Chem Phys 102(11), 4531-4537. https://doi.org/10.1063/1.469501

Luijten CCM, van Dongen MEH (1999) Nucleation at high pressure. I. theoretical considerations. J Chem Phys 111:8524-8534. https:// doi.org/10.1063/1.480193

Luijten CCM, van Hooy RGP, Janssen JWF, van Dongen MEH (1998) Multicomponent nucleation and droplet growth in natural gas. $\mathrm{J}$ Chem Phys 109:3553. https://doi.org/10.1063/1.476950

Luijten CCM, Peeters P, van Dongen MEH (1999) Nucleation at high pressure.II. Wave tube data and analysis. J Chem Phys 111:85358544. https://doi.org/10.1063/1.480194

Oxtoby DW, Kashchiev D (1994) A general relation between the nucleation work and the size of the nucleus in multicomponent nucleation. J Chem Phys 100(10), 7665-7671. https://doi.org/10. $1063 / 1.466859$

Oxtoby DW, Laaksonen A (1995) Some consequences of the nucleation theorem for binary fluids. J Chem Phys 102(17), 6846-6850. https://doi.org/10.1063/1.469121

Peters F (1983) A new method to measure homogeneous nucleation rates in shock tubes. Exp Fluids 1:143-148. https://doi.org/10. 1007/bf00272013

Strey R, Wagner PE, Viisanen Y (1994) The problem of measuring homogeneous nucleation rates and the molecular contents of nuclei: progress in the form of nucleation pulse measurements. J Phys Chem 98(32), 7748-7758. https://doi.org/10.1021/j1000 $83 \mathrm{a} 003$

Wagner PE (1985) A constant-angle mie scattering method (CAMS) for investigation of particle formation processes. Journal of colloid and interface science 105(2):456-467. https://doi.org/10.1016/ 0021-9797(85)90319-4

Wagner PE, Strey R (1981) Homogeneous nucleation rates of water vapor measured in a two-piston expansion chamber. J Phys Chem 85:2694-2698. https://doi.org/10.1021/j150618a026

Wilemski G (1995) The Kelvin equation and self-consistent nucleation theory. J Chem Phys 103:1119-1126. https://doi.org/10.1063/1. 469822

Wilson CTR, Thomson JJ (1897) Condensation of water vapour in the presence of dust-free air and other gases. Philos Trans R Soc London Ser A 189:265-307. https://doi.org/10.1098/rsta.1897.0011

Wölk J, Strey R (2001) Homogeneous nucleation of H2O and D2O in comparison: the isotope effect. J Phys Chem B 105:11683-11701. https://doi.org/10.1021/jp0115805

Wyslouzil BE, Wölk J (2016) Overview: Homogeneous nucleation from the vapor phase - the experimental science. J Chem Phys 145(21):211702. https://doi.org/10.1063/1.4962283

Publisher's Note Springer Nature remains neutral with regard to jurisdictional claims in published maps and institutional affiliations. 


\section{Authors and Affiliations}

\section{M. Campagna ${ }^{1}$ (D) J. Hrubý ${ }^{3} \cdot$ M. E. H. van Dongen ${ }^{2} \cdot$ D. M. J. Smeulders ${ }^{1}$}

1 Department of Mechanical Engineering, Eindhoven University of Technology, P.O. Box 513,

5600 MB Eindhoven, The Netherlands

2 Department of Applied Physics, Eindhoven University of Technology, P.O. Box 513, 5600 MB Eindhoven, The Netherlands 3 Institute of Thermomechanics of the Czech Academy
of Sciences, Dolejskova 5, CZ-182 00 Prague 8,

Czech Republic 